\title{
A teachable moment: Identifying hyperostosis frontalis interna in a gross anatomy cadaver laboratory
}

\author{
Julia N. DiSalle ${ }^{1}$, Alexis L. Novak ${ }^{1}$, Prasad S. Dalvi², Elisa M. Konieczko²
}

DiSalle JN, Novak AL, Dalvi PS, et al. A teachable moment: Identifying hyperostosis frontalis interna in a gross anatomy cadaver laboratory. Int J Anat Var. 2019;12(4): 35-39.

Hyperostosis frontalis interna (HFI) is a condition in which newly formed cancellous bone is deposited on the inner lamina of the cranium forming irregular thickening on the internal surface of the frontal bone. HFI is mostly considered as a benign entity; however, it may result in compression of brain tissue if the frontal bone is extensively hypertrophied. HFI may be associated with hormonal imbalance and/or neuropsychiatric abnormalities, such as behavioral disturbances and dementia. Although, the etiology of HFI remains largely unknown, there is marked female predominance as HFI has been frequently reported among postmenopausal elderly women, and is reported in males with severe hypogonadism and testicular atrophy. Here, we report a case of an 80 year-old woman who was diagnosed with Alzheimer's disease/vascular dementia at the time of death, and was found to have HFI with dural fusion during dissection in the human gross anatomy laboratory at Gannon Universityt.

Key Words: Hyperostosis frontalis interna (HFI); Frontal bone ossification; Dementia Hormonal disbalance; Cadavers

\section{INTRODUCTION}

$\mathrm{H}$ yperostosis frontalis interna (HFI) is a condition with limited bone remodeling of the neurocranium. In HFI, newly formed cancellous bone is deposited on the inner lamina of the cranium forming irregular thickening on the internal surface of the frontal bone. Such thickening has been long documented in the literature, beginning in the early 1700's with books written by Giovanni Batistta Morgagni, the father of anatomical pathology. This disease was further documented and detailed in the early $20^{\text {th }}$ century [1-3]. HFI is usually not the primary cause of death for an individual and is often found through scans ordered for other possible diseases [4]. Sometimes, however, the hypertrophy of the frontal bone can be extensive and may result in compression of brain tissue [5].

Despite the wide-ranging literature on HFI, the etiology of this disease remains largely unknown [6]. Some authors have suggested insanity or dementia may cause this physical change in the cranium $[1,5]$ while others have suggested hormonal causes [7-10] or metabolic disorders [4,11]. The literature is clear that there is marked female predominance in the epidemiology of this disorder, especially in post-menopausal elderly women [12-15]. However, HFI has also been reported in males with severe hypogonadism and testicular atrophy [9]. HFI is distinguished from other conditions, including acromegaly, Paget's disease and bone cancer of the skull $[4,10]$.

Gannon University teaches cadaver-based human gross anatomy to undergraduate college students in biology, chemistry, pre-physical therapy, and physician assistant majors [16]. These high volume high intensity courses involve both prosected cadavers and cadavers dissected by enrolled students [17]. One of the challenges of teaching cadaver-based anatomy is the extensive memorization students must achieve in order to be successful. Using pathologies discovered during dissection of the cadavers to supplement and enhance lecture material is one method used by course instructors to engage students and generate enthusiasm for learning complex material [18]. Here, we present a case of HFI that was not found in any of the previously dissected cadavers at the Gannon University human gross anatomy laboratory. This finding engaged instructors, teaching assistants and students, as they investigated the etiology, symptoms, and conditions of HFI. Gross anatomy laboratory instructors can use opportunities like this to engage students in anatomical and clinical discussions. These discussions enhance what is often a learning experience dominated by extensive memorization.

\section{CASE REPORT}

An 80 year-old woman who was diagnosed with Alzheimer's disease/vascular dementia at the time of death was found to have HFI with dural fusion to the calvarium during dissection in the Gannon University human gross anatomy laboratory (Figure 1). The examination of the internal side of the skull cap revealed that the inner table (lamina) of the calvarium was covered with large, irregular nodular bony thickening that was predominantly localized to the region of the frontal bones (Figure 1). Consequently, the frontal lobes were found to be compressed and subdural space was decreased by the nodular thickness of the frontal bones. The bony nodular thickening was not present on any part of the parietal or occipital bones. The thickness of the frontal bones was more marked and exceedingly dense than that of parietal or occipital bones, being apparently due to overgrowth of cancellous bony tissue (Figure 1). The diploë of the frontal bones was slightly reduced in amount. The dura mater was thickened and was adhered to the inner table of the skull and fused with, in particular, the frontal bone (Figure 2). The anterior end of falx cerebri contained a large bony plate, nearly a quarter of an inch thick and two inches long. The brain weighted less than the age-matching typical normal brain from another decedents without HFI (Table 1). The coronal sections of the brain described in this study revealed that both cerebral hemispheres were filled with a relatively larger amount of white matter composed of shallow sulci and atrophied gyri (Figure 3). In addition, the decedent's brain showed atrophy of the frontal gyri, and the cerebral ventricles were found to be reduced in size (Figure 4).

\section{DISCUSSION}

HFI was first described by Morgagni more than 300 years ago in the early 1700 's, and since then HFI has been associated with frontal headaches, psychoneurosis, obesity, pregnancy, acromegaly, virilism, hypertrichosis, diabetes, and other hormonal and metabolic disorders [19-22]. Based on these case reports and studies, HFI has been included in Morgagni's

${ }^{1}$ Department of General Medicine, Gannon University, Erie, PA, USA; ${ }^{2}$ Department of Biology, Gannon University, Erie, PA, USA

Correspondence: Prasad S. Dalvi, Department of Biology, Gannon University, Erie, PA, USA, Tel: 01-814-871-7651, Email: konieczk001@gannon.edu

Elisa M. Konieczko, Department of Biology, Gannon University, Erie, PA, USA, Tel: 01-814-871-5963, E-mail: dalvi001@gannon.edu

Received: July 14, 2019, Accepted: Sep 16, 2019, Published: Sep 23, 2019 org/licenses/by-nc/4.0/), which permits reuse, distribution and reproduction of the article, provided that the original work is properly cited and the reuse is restricted to noncommercial purposes. For commercial reuse, contact reprints@pulsus.com 
Dalvi P, et al.
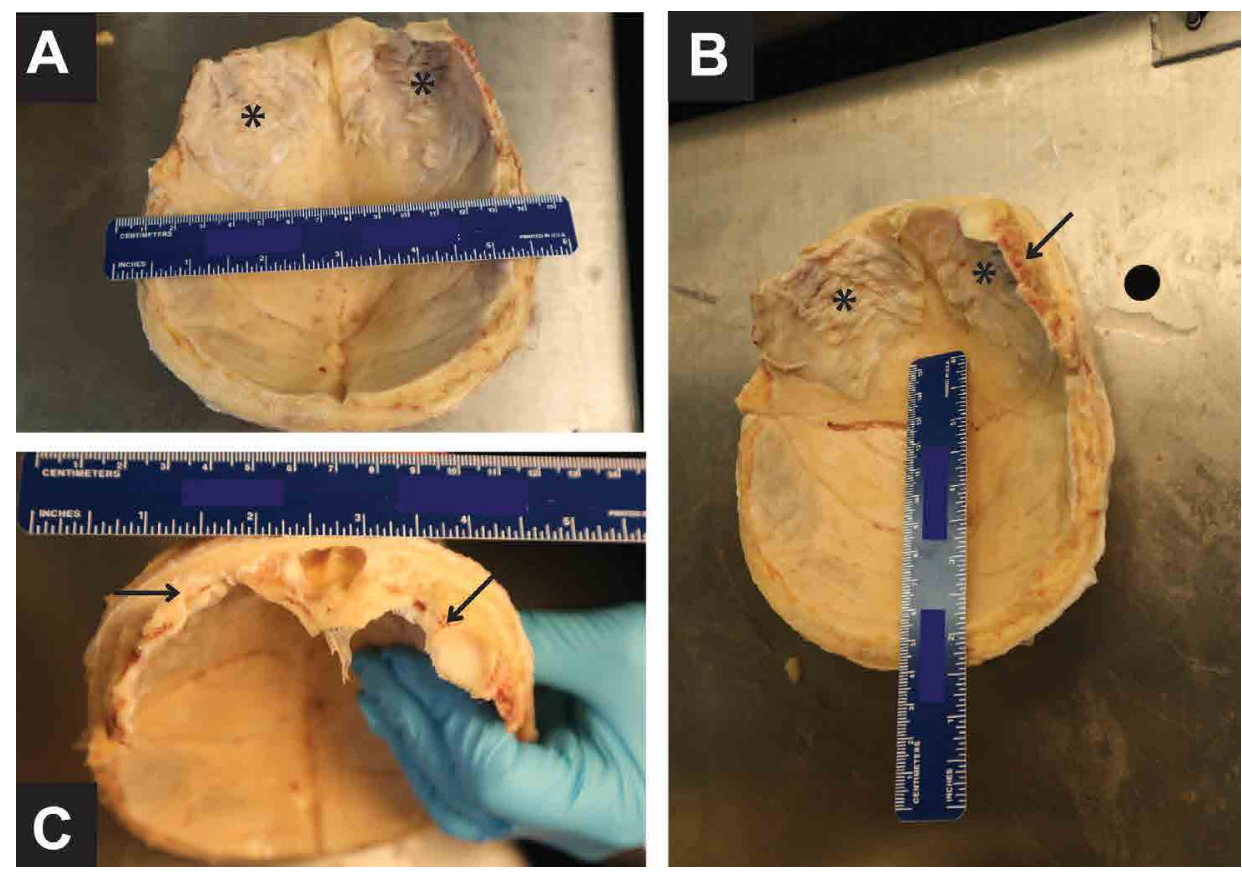

Figure 1) (A, B) Internal surface of the calvarium with a visible midline and newly formed cancellous bone deposited on either side of the inner lamina of the frontal bone forming irregular thickening on the internal surface (asterisks), (B, C) Large, irregular bony thickening on either side of the frontal bone (black arrows).
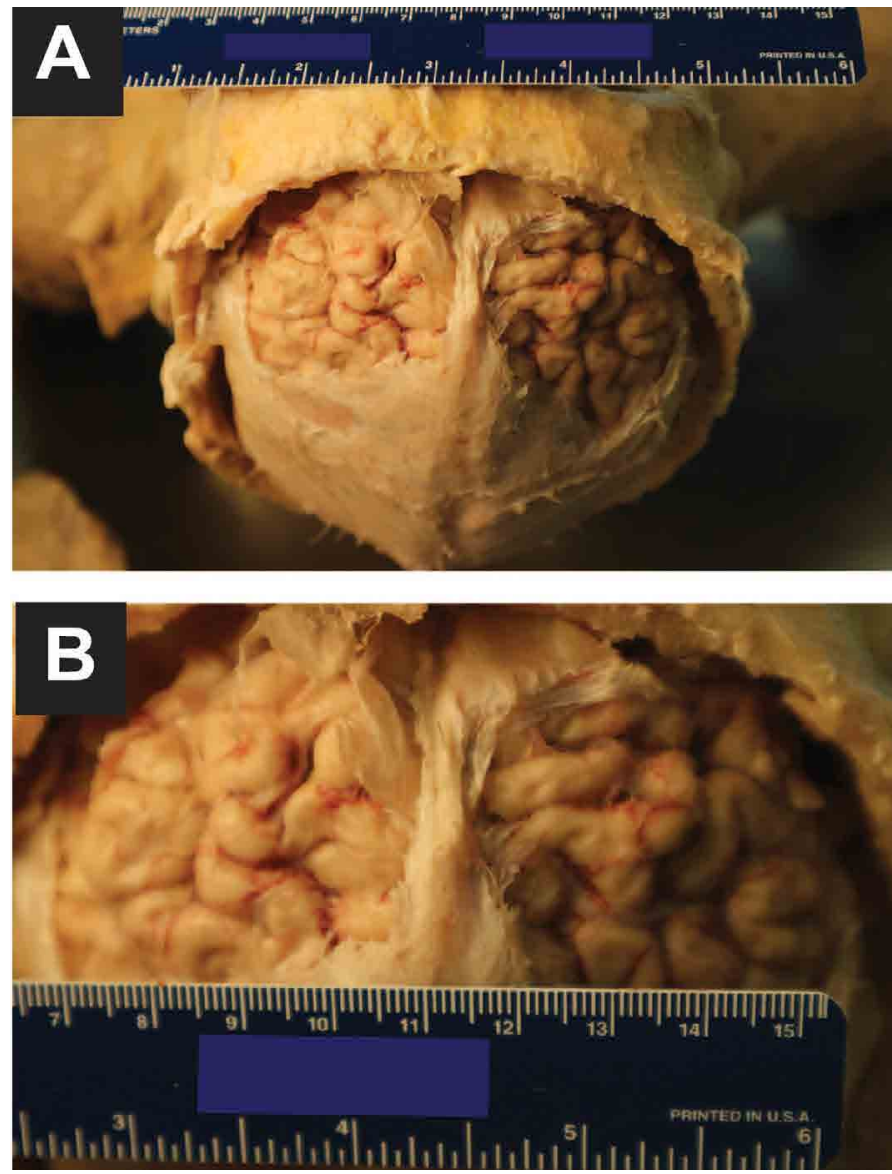

Figure 2) (A) The intact brain within the skull with the missing portion of dura mater over the frontal lobes as it was fused with the thickened inner lamina of the frontal bones and was removed with the calvarium during craniotomy, (B) Magnified image of the brain with the visibly intact gyri and sulci of the frontal lobe.

syndrome (HFI, obesity, virilism), Stewart-Morel syndrome (HFI, obesity, mental disturbances), and Troell-Junet syndrome (HFI, acromegaly, toxic goiter, and diabetes mellitus) $[19,22]$. However, at present, HFI is viewed as an independent condition representing a phenomenon itself rather than related to any specific syndrome as suggested in the past [12]. HFI
TABLE 1

Comparison of the weight of the decedent's brain with Hyperostosis Frontalis Interna (HFI) to the typical normal brains from other cadavers without HFI at the Human Gross Anatomy Laboratory at Gannon University.

\begin{tabular}{ccc}
\hline Brain & Weight $(\mathrm{g})$ & Presence of Dura Mater $(+$ or -$)$ \\
\hline Case study brain & 1046.31 & + \\
\hline Case study brain & 975.77 & - \\
\hline$\# 1$ & 1147.26 & + \\
\hline$\# 2$ & 1435.43 & + \\
\hline$\# 4$ & 1141.90 & + \\
\hline$\# 5$ & 1417.67 & + \\
\hline$\# 6$ & $1007.12^{*}$ & + \\
\hline$\# 7$ & $1121.97^{* *}$ & + \\
\hline$\# 8$ & 1401.24 *** & + \\
\hline$\# 9$ & 1315.59 & - \\
\hline$\# 10$ & 1367.86 & - \\
\hline$\# 11$ & $1458.64 \mathrm{~g}$ & - \\
\hline$\# 12$ & $1219.85 \mathrm{~g}$ & - \\
\hline$\# 13$ & 1216.73 & \\
\hline
\end{tabular}

* Did not have a cerebellum;

** half of the dura mater present;

*** with only falx cerebri

has been reported in $5-12 \%$ of the general population, but the magnitude of manifestation and frequency of HFI are much higher in the female population [12,23]. It is now accepted that HFI by itself does not cause a significant clinical disease and is usually an incidental finding, however, the bone growth can be exuberant and cause compression of the underlying brain tissue [5]. Past archeological investigations have shown that HFI was rarely present in historic populations regardless of geographical origin; however, on the other hand, the skulls from modern times have been found to develop HFI, predominantly in the post-menopausal female population [12]. Currently, the etiology of HFI remains uncertain, and therefore, several hypotheses have been proposed to explain HFI. One hypothesis proposes that during human evolution, a wider availability of food favored an increased metabolic rate which may have caused a higher incidence of HFI via increased levels of leptin, a satiety peptide that increases sympathetic tone and energy expenditure [24]. Another explanation for detecting HFI more 

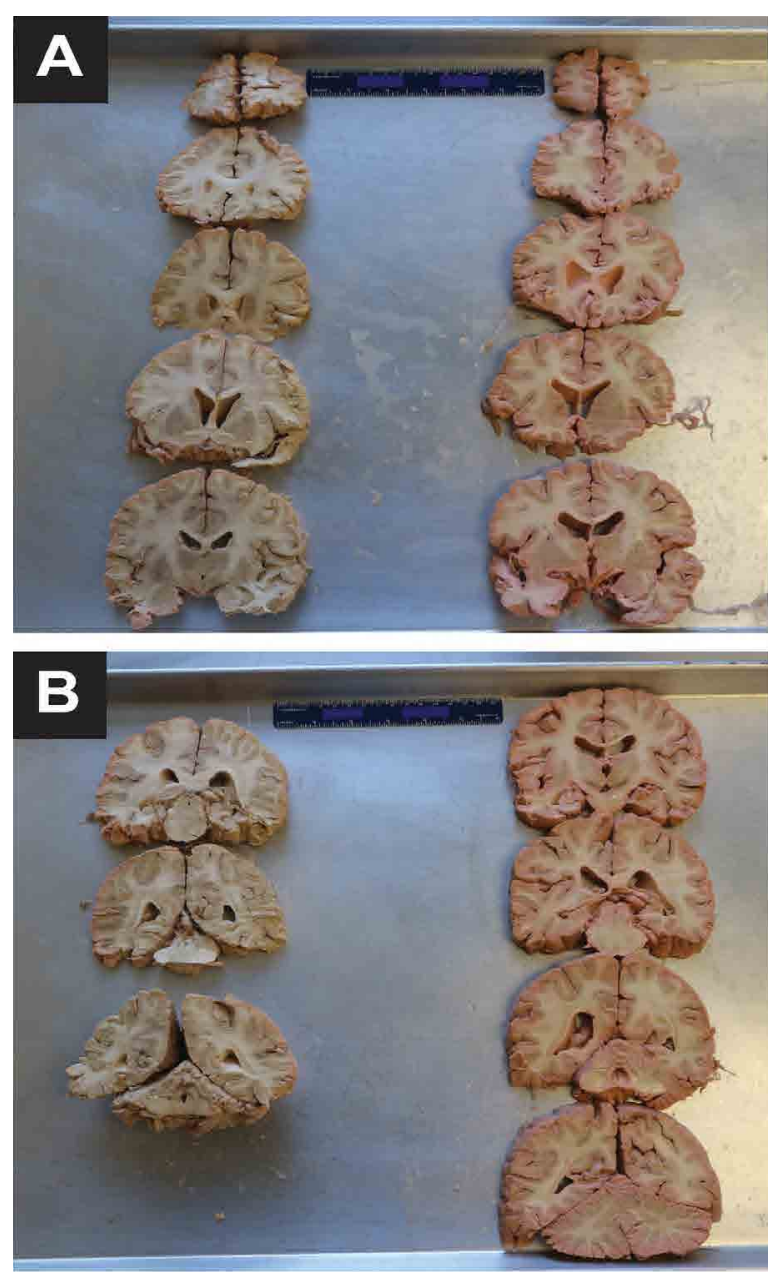

Figure 3) (A, B) Comparison of the serial coronal sections of the decedent's brain on the left side of both images to a typical normal brain on the right side. The sections of the decedent's brain show relatively larger amount of white mater.
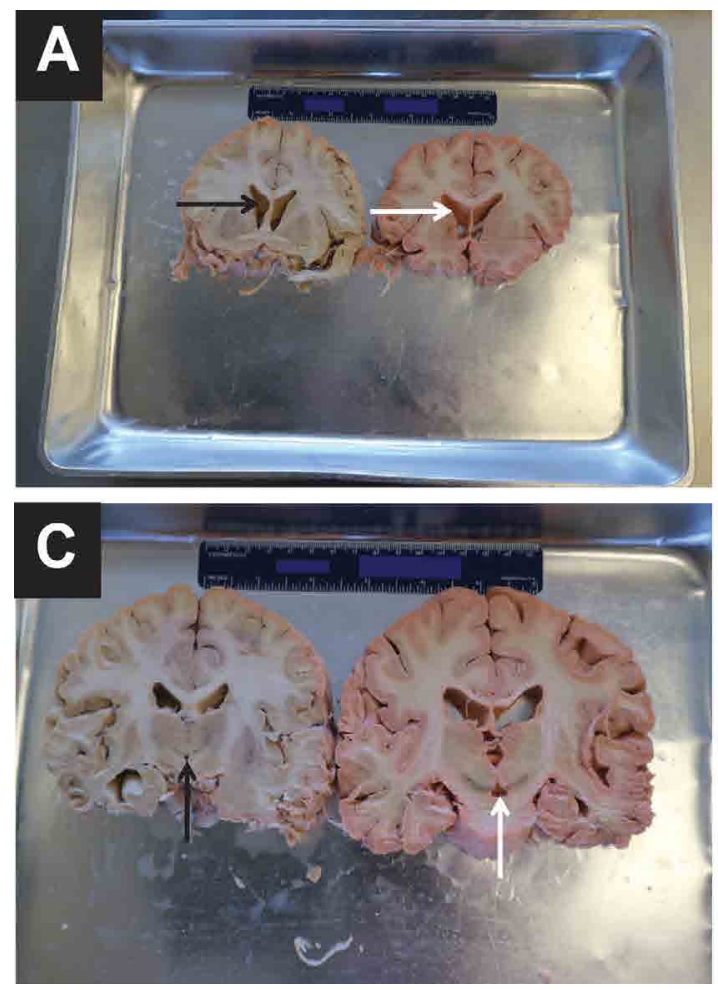

frequently in elderly population is that greater longevity has allowed HFI to become more prevalent and detectable in the aging population [15]. Another hypothesis is that prolonged high levels of estrogen during the reproductive period of life may be the primary triggering factor contributing to the greater frequencies of HFI seen in post-menopausal females [25], and therefore, the incidence in the female population has been reported to be much higher than in male population [12]. While HFI is found predominantly in females, it is not a purely female phenomenon. Males with hormonal irregularities, such as atrophic testis and/or feminism were found to have HFI of variable severity $[9,12]$. On the other hand, it has been shown that HFI is related to elevated androgen levels in pre-menopausal women [26]. At present, the most acceptable hypothesis regarding HFI pathogenesis is hormonal imbalance of the gonads.

HFI is usually not the primary cause of death for an individual and is often found through scans ordered for other possible diseases, and therefore, upon recognition of prominent HFI in imaging, it is important to distinguish it from other pathologic processes, such as leptomeningeal metastasis, hemorrhage, or infection [5]. While HFI is a benign process, prominent HFI may cause compression of the underlying soft tissues, inflammation and irritation of the meninges and pressure atrophy of the brain, for which surgical decompression is the treatment [27]. Cases of HFI, with or without neuropsychiatric disorders, have been reported by several authors $[1,4,5,9,11]$. These studies report that the obesity, diabetes, dyslipidemia and other metabolic disturbances, hormonal imbalance, post-menopausal state, and female gender are considered be the risk factors of HFI. The only known risk

\section{TABLE 2}

Review of literature for the brain weight observed in the cases of HFI with neurological symptoms.

\begin{tabular}{cccc}
\hline Gender & $\begin{array}{c}\text { Age } \\
\text { (Years) }\end{array}$ & Pathology & $\begin{array}{c}\text { Post-mortem brain } \\
\text { weight }(\mathrm{g})\end{array}$ \\
\hline Female & 48 & Melancholia, Loss of Memory & 1150 \\
\hline Female & 47 & $\begin{array}{c}\text { Dementia, Disorientation in } \\
\text { time and place }\end{array}$ & 1247 \\
\hline Female & 62 & $\begin{array}{c}\text { Recurrent melancholia, } \\
\text { dementia }\end{array}$ & 1169 \\
\hline
\end{tabular}
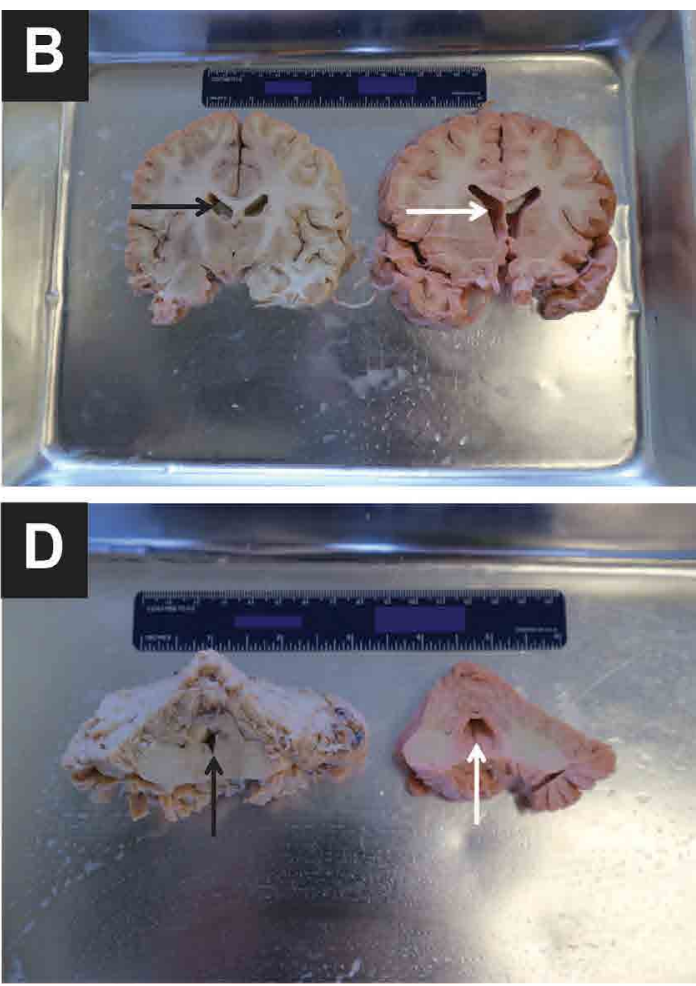

Figure 4) (A-D) Comparison of the serial coronal sections of the decedent's brain on the left side to a typical brain on the right side. (A, B) The decedent's brain shows reduced volume of the lateral ventricles (black arrows) compared to the typical normal brain (white arrows), (C) The decedent's brain shows decreased size of the third ventricle (black arrow) compared to the typical normal brain (white arrow), (D) The decedent's brain shows reduced volume of the fourth ventricle (black arrow) compared to the typical normal brain (white arrow). 
factors for the development of HFI in the present case study were the postmenopausal age and the female gender of the decadent. Gross examination of the decadent's inner table of the skull showed the characteristic bony nodular overgrowth of HFI that is not typically seen in other disease entities, such as rickets, acromegaly, Paget's disease, or leontiasis ossea [10]. Most of the above-mentioned HFI case studies have focused on the internal surface of the frontal bone with no mention of any changes that could have occurred in the brain due to possible decreased intracranial volume. Only one study provided information about the brain weight and associated structural changes (Table 2) [1]. In the present case study, we measured the brain weight (Table 1) and compared it with another typical normal age-matching brain without HFI (Figures 3 and 4). The brain weight of the decadent was found to be decreased (Table 1). The presence of decreased ventricular volume suggests the compression of the brain tissue and a decrease in brain volume that could have a major clinical significance as it may indicate the beginning of degenerative processes of the brain. Indeed, the decedent was diagnosed with Alzheimer's disease/vascular dementia at the time of death. Among other changes that confirm the neurodegenerative process, we observed that the frontal gyri were atrophied, and both cerebral hemispheres had increased white matter (Figures 3 and 4).

The first human dissection was recorded around $300 \mathrm{BC}$ [28], and human cadavers have been used as an educational tool since the $16^{\text {th }}$ or $17^{\text {th }}$ century [29]. However, there is an ongoing debate about the practicality and usefulness of cadavers as an educational tool [30]. With the advancement in technology, the way anatomy is taught is changing, and the use of cadavers may become obsolete [31,32]. Computer technology has provided an alternative to both cadavers and textbook images, and is becoming popular $[31,32]$. Despite this, many academic institutions continue to believe that cadaveric dissection is still the best way to educate students about the complexity of the human body and continue to use the human cadavers in the classroom [33]. Supporters for the use of cadavers argue that human cadavers not only can provide the visual and tactile experiences necessary to learn and apply anatomical knowledge, but also provide in situ information and details about the complex human body structures with or without any existing pathology [34]. At Gannon University, cadaver-based human gross anatomy is taught to undergraduate college students in biology, chemistry, pre-physical therapy, and physician assistant majors [16]. Quite often we come across cadavers with variability in anatomical structures and pathologies. The enrolled students are very interested in these anomalies and diseases, and ask questions about them. Often, the undergraduate students are quite passive in their learning. This opportunity clearly engages the students and makes them active learners. It allows the instructors and teaching assistants to provide additional information about the anomalies and pathologies seen, and we incorporate this material in course assessments.

The present case of HFI was the first case found at the human gross anatomy laboratory at Gannon University within past 20 years, and therefore, this finding was quite novel to the instructors, teaching assistants and students. Gross anatomy laboratory instructors can use similar cases to engage students in anatomical and clinical discussions that can promote longterm retention of pathological information useful for the future clinical and health professionals. Medical professionals of all expertise believe that cadaveric dissection enables learning anatomy with relevant clinical correlates, and complemented with other innovative learning methods lays strong foundation critical to development of clinical skills [34].

\section{CONCLUSION}

Endocrine imbalance, metabolic disorders, and/or neuropsychiatric abnormalities may be responsible for the development of HFI in some cases. The epidemiology of HFI indicates marked female predominance. HFI is usually not the primary cause of death for an individual and is often found through scans ordered for other possible diseases. Although the etiopathogenesis of HFI is not quite clear, it is important to correlate its development to an underlying manifestation of an endocrine, metabolic and neuropsychiatric condition. Gross anatomy laboratory instructors can use similar cases to engage students in anatomical and clinical discussions through active learning strategies that can promote long-term retention of pathological information useful for the future clinical and health professionals. These discussions enhance what is often a learning experience dominated by extensive memorization.

\section{ACKNOWLEDGEMENTS}

The authors wish to thank the biology department of Gannon University for its financial support of the publication of this article. The authors thank the Wright State Anatomical Gift program and the Booneshaft School of Medicine for the permission to publish this case study.

\section{REFERENCES}

1. Stewart RM. Localised Cranial Hyperostosis in the Insane. J Neurol Psychopathol. 1928;8:321-31.

2. Moore S. Calvarial hyperostosis and the accompanying symptom complex. Arch Neurol Psychiatry. 1936;35:975-81.

3. Knies PT, Le Fever HE. Metabolic craniopathy: hyperostosis frontalis interna. Ann Intern Med. 1941;14:1858-92.

4. She R, Szakacs J. Hyperostosis frontalis interna: case report and review of literature. Ann Clin Lab Sci. 2004;34:206-8.

5. Chaljub G, Johnson RF, Johnson RF Jr, et al. Unusually exuberant hyperostosis frontalis interna: MRI. Neuroradiology. 1999;41:44-5.

6. Raikos A, Paraskevas GK, Yusuf F, et al. Etiopathogenesis of hyperostosis frontalis interna: a mystery still. Ann Anat. 2011;193:453-8.

7. Goodman DH, Eragan A. Gout, myxedema, and hyperostosis frontalis interna. Report of case in Negro woman. JAMA. 1960;173:1734-5.

8. Pawlikowski M, Komorowski J. Hyperostosis frontalis, galactorrhoea/ hyperprolactinaemia, and Morgagni-Stewart-Morel syndrome. Lancet. $1983 ; 1: 474$

9. Yamakawa K, Mizutani K, Takahashi M, et al. Hyperostosis frontalis interna associated with hypogonadism in an elderly man. Age Ageing. 2006;35:202-3

10. Fulton JD, Shand J, Ritchie D, et al. Hyperostosis frontalis interna, acromegaly and hyperprolactinaemia. Postgrad Med J. 1990;66:16-9.

11. Sohmiya M, Tanaka M, Aihara Y, et al. Hyperostosis cranii in the elderly with various clinical symptoms. Geriatr Gerontol Int. 2004;4:59-63.

12. Hershkovitz I, Greenwald C, Rothschild BM, et al. Hyperostosis frontalis interna: an anthropological perspective. Am J Phys Anthropol. 1999; 109:303-25

13. Devriendt W, Piercecchi-Marti MD, Adalian P, et al. Hyperostosis frontalis interna: forensic issues. J Forensic Sci. 2005;50:143-6.

14. May H, Mali Y, Dar G, et al. Intracranial volume, cranial thickness, and hyperostosis frontalis interna in the elderly. Am J Hum Biol. 2012;24:812-9.

15. Cvetkovic D, Nikolic S, Brkovic V, et al. Hyperostosis frontalis interna as an age-related phenomenon - Differences between males and females and possible use in identification. J Forensic Sci Society. 2019;59:172-6.

16. Konieczko EM, Mattinson CE. Eighty Students, Five Cadavers, and Two Professors: Innovative Teaching Practices in an Undergraduate Human Gross Anatomy Course. HAPS Educator. 2017;21:95-100.

17. Mattinson CE, Konieczko EM. A Method for Maximizing Dissection Experience with a Minimal Number of Cadavers in an Undergraduate Human Gross Anatomy Laboratory Course. HAPS Educator. 2017;21:43-8.

18. Aziz MA, McKenzie JC, Wilson JS, et al. The human cadaver in the age of biomedical informatics. Anat Rec. 2002;269:20-32.

19. Dann S. Metabolic craniopathy: a review of the literature with report of a case with diabetes insipidus. Ann Intern Med. 1951;34:163-202.

20.Salmi A, Voutilainen A, Holsti IR, et al. Hyperostosis cranii in a normal population. Am J Roentgenol Radium Ther Nucl Med. 1962;87:1032-40.

21. Crispin JC, Alcocer-Varela J. Rheumatologic manifestations of diabetes mellitus. Am J Med. 2003;114:753-7.

22. Moore S. The Troell-Junet syndrome. Acta Radiol. 1953;39:485-93.

23. Nikolic S, Djonic D, Zivkovic V, et al. Rate of occurrence, gross 
appearance, and age relation of hyperostosis frontalis interna in females: a prospective autopsy study. Am J Forensic Med Pathol. 2010;31:205-7.

24. Ruhli FJ, Henneberg M. Are hyperostosis frontalis interna and leptin linked? A hypothetical approach about hormonal influence on human microevolution. Med Hypotheses. 2002;58:378-81.

25. Korenman SG. Oestrogen window hypothesis of the aetiology of breast cancer. Lancet. 1980;1:700-1.

26. Kollin E, Feher T. Androgens, bone mineral content and hyperostosis frontalis interna in pre-menopausal women. Exp Clin Endocrinol. $1986 ; 87: 211-4$.

27. Hasegawa T, Ito H, Yamamoto S, et al. Unilateral hyperostosis frontalis interna. Case report. J Neurosurg. 1983;59:710-3.

28. von Staden H. The discovery of the body: human dissection and its cultural contexts in ancient Greece. Yale J Biol Med. 1992;65:223-41.
29. Klestinec C. A history of anatomy theaters in sixteenth-century Padua. J Hist Med Allied Sci. 2004;59:375-412.

30. Tam MDBS, Hart AR, Williams S, et al. Is learning anatomy facilitated by computer-aided learning? A review of the literature. J Medical Teacher. 2009;31:e393-e6.

31. Biasutto SN, Caussa LI, Criado del Rio LE. Teaching anatomy: cadavers vs. computers?. Ann Anat. 2006;188:187-90.

32. Patel SB, Mauro D, Fenn J, et al. Is dissection the only way to learn anatomy? Thoughts from students at a non-dissecting based medical school. Perspect Med Educ. 2015;4:259-60.

33. Memon I. Cadaver Dissection Is Obsolete in Medical Training! A Misinterpreted Notion. Med Princ Pract. 2018;27:201-10.

34. Ghosh SK. Cadaveric dissection as an educational tool for anatomical sciences in the 21st century. Anat Sci Educ. 2017;10:286-99. 